\title{
CATALOGING OF THE DIGITIZED POSS-II:
}

\section{INITIAL SCIENTIFIC RESULTS}

\author{
S.G. DJORGOVSKI ${ }^{1}$, R.R. De CARVALHO ${ }^{1,2}$, R.R. GAL ${ }^{1}$, \\ M.A. PAHRE ${ }^{1}$, R. SCARAMELLA ${ }^{3}$ AND G. LONGO ${ }^{4}$ \\ ${ }^{1}$ Palomar Observatory, Caltech, Pasadena, CA 91125, USA \\ ${ }^{2}$ Observatorio Nacional, CNPq, 20921 Rio de Janeiro, Brasil \\ ${ }^{3}$ Osservatorio Astr. di Roma, I-00040 Monteporzio, Italy \\ ${ }^{4}$ Osservatorio Astr. di Capodimonte, I-80131 Napoli, Italy
}

\section{The Survey}

The Second Palomar Sky Survey (POSS-II) is now nearing completion. It will cover the entire northern sky with 894 fields $\left(6.5^{\circ}\right.$ square) at $5^{\circ}$ spacings, with no gaps in the coverage. Plates are taken in three bands: IIIa-J + GG395, $\lambda_{\text {eff }} \sim 480 \mathrm{~nm}$; IIIa-F + RG610, $\lambda_{\text {eff }} \sim 650 \mathrm{~nm}$; and IV-N + RG9, $\lambda_{e f f} \sim 850 \mathrm{~nm}$. Typical limiting magnitudes reached are $B_{J} \sim 22.5, R_{F} \sim 20.8$, and $I_{N} \sim 19.5$, i.e., $\sim 1^{m}-1.5^{m}$ deeper than the POSS-I. The image quality is improved relative to the POSS-I, and is comparable to the southern photographic sky surveys. For more details, see Reid et al. (1987), and Reid \& Djorgovski (1993).

The plates are being digitized at STScI, using modified PDS scanners (see the papers by McLean, Lasker, et al. in this volume). Plates are scanned with 15 -micron $(1.0 \mathrm{arcsec})$ pixels, in rasters of 23,040 square, giving $\sim 1 \mathrm{~GB} /$ plate, or $\sim 3 \mathrm{~TB}$ of pixel data total for the entire digital survey (DPOSS). Preliminary astrometric solutions are good to $\sim 0.5$ arcsec, and will get better soon. Completion of the scanning should follow closely the completion of the plate taking, hopefully by mid-1998. The plates are also digitized independently at USNOFS by Monet et al.

There is a major ongoing effort at Caltech to process and calibrate the scans, while at the same time catalog and classify all objects detected down to the survey limit. We are using SKICAT, a novel software system developed for this purpose (Weir et al. 1993ab, 1994, 1995abc; Djorgovski et al. 1994; Fayyad et al. 1996). SKICAT incorporates some standard astro- 
nomical image processing packages, commercial Sybase DBMS, as well as a number of artificial intelligence (AI) and machine learning based modules.

As of late 1996, some $15 \%$ of the entire survey area has been processed, but this work will soon speed up considerably. We have started a collaborative effort between the Caltech DPOSS group and the Observatories of Rome and Naples (Italy) to complete the DPOSS processing in a timely manner (project CRONA). Data processing pipelines are now being set up at both Italian sites. Observatorio Nacional in Rio de Janeiro (Brasil) will join the consortium in 1997.

The resulting Palomar-Norris Sky Catalog (PNSC) will contain all objects down to an equivalent limiting magnitude of $B_{J} \sim 22^{m}$, with stargalaxy classification accurate to $90 \%$ or better, down to $B_{J} \sim 21^{m}$. The PNSC is expected to contain $>50$ million galaxies, and $>2$ billion stars, including $\sim 10^{5}$ quasars. We note that the size of the DPOSS data set, in terms of the bits, numbers of sources, and resolution elements, is $\approx 1,000 \times$ the entire IRAS data set, and is $\approx 0.1 \times$ the anticipated SDSS data set.

We will publish the catalogs as soon as the validation tests are complete, and the funding allows it, via computer networks and other suitable media. The anticipated project completion timescale is $\sim 3$ years.

\section{The Data}

A particular strength of SKICAT is the star-galaxy classification, which uses artificial induction decision tree techniques. By using these methods, and using superior CCD data to train the AI object classifiers, we are able to achieve classification accuracy of $90 \%$ or better down to $\sim 1^{m}$ above the plate detection limit; traditional techniques achieve comparable accuracy typically only $\sim 2^{m}$ above it. This effectively triples the number of usable objects for most scientific applications of these data, since in most cases one wants either stellar objects or galaxies.

Future technical developments include an improved treatment of very bright and/or extended objects, optimization of the object measurement module for crowded regions (e.g., low Galactic latitudes), better structuring of the catalog database for efficient access and manipulation, and testing and implementation of novel methods for data exploration, including unsupervised classifiers and clustering analysis algorithms, etc. Some initial results have been presented by de Carvalho et al. (1995).

An extensive CCD calibration effort is now underway at the Palomar 60 -inch telescope, and we expect it to expand to other sites soon. The data are calibrated in the Gunn gri system. We obtain 2 CCD images per sky survey field, sometimes more. Usually these CCD images are used both for magnitude zero-point calibrations, and for training of automated 
star-galaxy classifiers. In addition to the CCD calibrations, we use heavily smoothed sky measurements from the plate scans themselves (after the object removal) to "flatfield" away the telescope vignetting effects and the individual plate emulsion sensitivity variations.

As a result, we have demonstrated an unprecedented photometric stability and accuracy for this type of photographic plate material (Weir et al. 1995a). We have performed tests using both CCD sequences and plate overlaps, and find that our magnitude zero-points are stable to within a few percent, across the plates, between adjacent plates, and across the individual plates. Typical r.m.s. in the magnitude zero-points between different plates is $0.015^{m}-0.045^{m}$ in the $r$ band, slightly worse in the $g$ band, perhaps due to the larger color terms in the $\mathrm{J} / g$ calibration. Keeping the systematic magnitude zero-point errors below $10 \%$ is essential for many scientific applications of these data.

This may be best illustrated in the internal consistency of galaxy counts from sets of adjacent POSS-II plates. We compared the counts published by Picard (1991), who used COSMOS machine scans processed in a traditional way, with the counts from Weir et al. (1995a), who used DPOSS scans processed with SKICAT. While Picard has seen large plate-to-plate variations (a factor of 2 , or more) in number counts at a given magnitude, Weir finds excellent agreement, to within the Poissonian errors, and reaches a magnitude deeper; yet both used the same kind of plate material.

Median random magnitude errors for stellar objects in all three bands start around $0.05^{m}$ at the bright end, and increase to $\sim 0.25^{m}$ at $g_{\text {lim }} \approx$ $22^{m}, \sim 0.20^{m}$ at $r_{\text {lim }} \approx 21.5^{m}$, and $\sim 0.25^{m}$ at $i_{\text {lim }} \approx 20^{m}$. For galaxies, these errors are typically higher by about $50 \%$ at a given magnitude.

\section{Some Initial Scientific Applications}

This large new database should be a fertile ground for numerous scientific investigations for years to come. The nature of the data dictates its uses: these images are not very deep by modern standards, but they do cover uniformly a very large solid angle. In addition to the obvious applications such as large-scale optical identifications of sources from other wavelengths (e.g., radio, X-ray, IR), there are two kinds of studies which can be pursued very effectively with data sets of this size:

(1) Statistical astronomy studies, where the sheer large numbers of detected sources tighten the statistical errors and allow for more model parameters to be constrained meaningfully by the data.

(2) Searches for rare types of objects. For example, at intermediate Galactic latitudes, about one in a million stellar objects down to $r \approx 19.5^{m}$ is a quasar at $z>4$, although we can find such quasars very efficiently. 
We have already started a number of scientific projects using DPOSS, which not also serve as scientific verification tests of the data, but have helped us catch some errors and improve and control the data quality.

Galaxy counts and colors in 3 bands from DPOSS can serve as a baseline for deeper galaxy counts and a consistency check for galaxy evolution models. Our initial results (Weir et al. 1995a) show a good agreement with simple models of weak galaxy evolution (e.g., Koo et al. 1995) at low redshifts, $z \sim 0.1-0.3$. We are now expanding this work to a much larger area, to average over the local large-scale structure variations. Our galaxy catalogs have been used as input for redshift surveys down to $\sim 21^{m}$, e.g., in the Palomar-Norris survey (Small et al. 1997). Several other groups also plan to use them for their own redshift surveys.

Galaxy $n$-point correlation functions and power spectra of galaxy clustering provide useful constraints of the CDM and other scenarios of large scale structure. Our preliminary results from a limited area near the NGP (Brainerd et al. 1995; and in preparation) indicate that there is less power at large scales than was found by the APM group (Maddox et al. 1989) in their southern survey. We suspect that field-to-field magnitude zero-point calibration errors and errors in star-galaxy separation in the APM data may account for this discrepancy. High quality, uniform calibrations are absolutely essential for this task. We are now also starting to explore the correlations of our galaxy counts with H I, IRAS, and DIRBE maps in order to better quantify the foreground Galactic extinction. We expect to generate extinction maps superior to those now commonly used.

We are now starting a project to generate an objectively defined, statistically well defined catalog of rich clusters of galaxies. We estimate that eventually we will have a catalog of as many as 20,000 rich clusters of galaxies at high Galactic latitudes in the northern sky. Their median redshift is estimated to be $\langle z\rangle \sim 0.2$, and perhaps reaching as high as $\langle z\rangle \sim 0.5$.

There are many cosmological uses for rich clusters of galaxies. They provide useful constraints for theories of large-scale structure formation and evolution, and represent valuable (possibly coeval) samples of galaxies to study their evolution in dense environments. Studies of the cluster twopoint correlation function are a powerful probe of large-scale structure, and the scenarios of its formation. Correlations between optically and Xray selected clusters are also of considerable scientific interest. Most of the studies to date have been limited by the statistical quality of the available cluster samples. For instance, the subjective nature of the Abell catalog has been widely recognized as its major limitation. Still, many far-reaching cosmological conclusions have been drawn from it. There is thus a real need to generate well-defined, objective catalogs of galaxy clusters and groups, with well understood selection criteria and completeness. 
We use only objects classified as galaxies in DPOSS catalogs, down to $r=19.6^{m}$, where the accuracy of object classifications is $>90 \%$. We then use colors for selection of the candidate cluster galaxies: early-type galaxies should better delineate high-density regions. Next we apply the adaptive kernel method to create the surface density maps. Its major advantage is that it uses a two-step process which smooths well the low density regions, and at the same time leaves the high density peaks nearly unaffected. Finally we evaluate the statistical significance of the density peaks using a bootstrap technique. Typically we set our threshold at a $4.5 \sigma$ level, where we successfully recover all of the known Abell clusters of richness class 0 and higher, and also find a large number of new cluster candidates which were apparently missed by Abell. We have also started spectroscopic follow-up of our cluster candidates at the Palomar 200-inch telescope.

Another ongoing project is a survey for luminous quasars at $z>4$. Quasars at $z>4$ are valuable probes of the early universe, galaxy formation, and the physics and evolution of the intergalactic medium at large redshifts. The continuum drop across the Ly $\alpha$ line gives these objects a distinctive color signature: extremely red in $(g-r)$, yet blue in $(r-i)$, thus standing away from the stellar sequence in the color space. Traditionally, the major contaminant in this type of work are red galaxies. Our superior star-galaxy classification leads to a manageable number of color-selected candidates, and an efficient spectroscopic follow-up. As of late 1996, over 25 new $z>4$ quasars have been discovered. Images are available to other astronomers for their studies as soon as the data are reduced.

Our initial results (Kennefick et al. 1995ab) are the best estimates to date of the bright end of the quasar luminosity function (QLF) at $z>4$, and are in excellent agreement with the fainter QLF evaluated by Schmidt et al. (1995). We have thus confirmed the decline in the comoving number density of bright quasars at $z>4$. There are also some intriguing hints of possible primordial large-scale structure as marked by these quasars. However, much more data is needed to check this result.

We have also stared optical identifications of thousands of VLA FIRST radio sources (Becker et al. 1995). Our preliminary results indicate that there are $\sim 400$ compact radio source IDs per DPOSS field, and we expect a comparable number of resolved source IDs. Among the first 10 red stellarlike IDs we have observed spectroscopically in May 1996, we discovered a quasar at $z=4.36, \mathrm{VF} 141045+340909$. We estimate that a few tens of $z>4$ quasars will be found in the course of this work. Eventually, we expect to have $>10^{5}$ IDs for the VLA FIRST sources, plus many more from other surveys.

In the area of statistical gravitational lensing studies, we have explored the possibility of microlensing of quasars, by looking for a possible excess 
of foreground galaxies near lines of sight to apparently bright, high- $z$ QSOs from flux-limited samples (Barton et al., in prep:). We find, at most, a modest excess, roughly as expected from theory, in contrast to some previous claims which used similar data (e.g., Webster et al. 1988). We are also planning to use our galaxy counts to explore the possible lensing magnification of background AGN by foreground large scale structure, as proposed, e.g., by Bartelmann \& Schneider (1994).

Other extragalactic projects now planned include a catalog of $\sim 10^{5}$ brightest galaxies in the northern sky, with a quantitative surface photometry and morphological information, automated searches for low surface brightness galaxies, an archival search for supernovæ from plate overlaps, derivation of photometric redshift estimators for galaxies, automated optical identifications of IR and X-ray sources, and so on.

Galactic astronomy should not be neglected. Star counts as a function of magnitudes, colors, position, and eventually proper motions as well, fitted over the entire northern sky at once, would provide unprecedented discrimination between different Galactic structure models, and constraints on their parameters. With $\sim 2 \times 10^{9}$ stars, such studies would present a major advance over similar efforts done in the past.

We can also search for stars with unusual colors or variability. We have started a search for stars at the bottom of the main sequence and field brown dwarf candidates, using colors: anything with $(r-i)>2.5$ should be interesting. At high Galactic latitudes, about one star in a million is that red, down to the conservative limit used so far $\left(r<19.5^{m}\right)$. Such a survey can be made much more powerful with the addition of IR data.

The same techniques we use to search for galaxy clusters can then be applied to our star catalogs, in an objective and automated search for sparse globulars in the Galactic halo, tidal disruption tails of former clusters, and possibly even new dwarf spheroidals in the Local Group (recall the Sextans dwarf, found using similar data by Irwin et al. 1990).

\section{Concluding Remarks}

These, and other studies now started or planned, should produce many interesting and useful new results in the years to come. Availability of large data sets such as DPOSS over the Net or through other suitable mechanisms would also enable astronomers and their students anywhere, even if they are far from the major research centers or without an access to large telescopes, to do some first-rate observational science. This new abundance of good data may profoundly change the sociology of astronomy.

Nor should we discount serendipity: With a data set as large as DPOSS, there is even an exciting possibility of discovering some heretofore unknown 
types of objects or phenomena, whose rarity would have made them escape the astronomers' notice so far.

This is a foretaste of things to come: with DPOSS, GSC-II, and surveys to follow (e.g., SDSS, 2MASS, etc.), we are changing the very concept of an astronomical catalog, into a living, permanently evolving data set, which must come along with adequate tools for its exploration. We will need to learn new skills, develop new data mining and exploration tools (including $\mathrm{AI}$ and machine learning techniques), new data structuring paradigms and standards, and above all, learn to ask new kinds of astronomical questions.

We are grateful to our many collaborators, including Nick Weir, Joe Roden, Julia Kennefick, Tereasa Brainerd, Usama Fayyad, Jeremy Darling, Vandana Desai, Emil Kartalov, Paul Stolorz, Alex Gray, Daniel Stern, and Isobel Hook; to Neill Reid, Jean Mueller, and others in the POSS-II survey team; to Barry Lasker, Brian McLean, and others in the digitization team at STScI; and to Massimo Capaccioli, Roberto Buonanno, and other CRONA-ies. This work was supported at Caltech by the NSF PYI award AST-9157412, grants from NASA, the Bressler Foundation, and Palomar Observatory. This paper is the CRONA Contribution No. 1.

\section{References}

Bartelmann, M., \& Schneider, P. 1994, Astron.Astrophys., 284, 1

Becker, R., White, R., \& Helfand, D. 1995, Astrophys.J., 450, 559

Brainerd, T., de Carvalho, R., \& Djorgovski, S. 1995, Bull.Am.Astron.Soc., 27, 1364

de Carvalho, et al. 1995, Astron. Soc. Pacific Conf. Series, 77, 272

Djorgovski, S., Weir, N. \& Fayyad, U. 1994, Astron. Soc. Pacific Conf. Series, 61, 195

Fayyad, U., Djorgovski, S.G. \& Weir, N. 1996, in Advances in Knowledge Discovery and Data Mining, eds. U. Fayyad et al., (Boston: AAAI/MIT Press), 471

Irwin, M., et al. 1990, Mon.Not.R.astron.Soc, 244, 16P

Kennefick, et al. 1995a, Astron.J., 110, 78

Kennefick, J.D., Djorgovski, S.G. \& de Carvalho, R. 1995b, Astron.J., 110, 2553

Koo, D., Gronwall, C., \& Bruzual, G. 1995, Astrophys.J., 440, L1

Maddox, S., et al. 1989, Mon.Not.R.astron.Soc., 242, 43P

Reid, I.N., et al. 1987, Publ.Astron.Soc.Pacific, 103, 661

Reid, I.N., \& Djorgovski, S. 1993, Astron. Soc. Pacific Conf. Series, 43, 125

Schmidt, M., Schneider, D. \& Gunn, J. 1995, Astron.J., 110, 68

Small, T., Sargent, W., \& Hamilton, D. 1997, Astrophys.J.Supp., in press

Webster, R., Hewett, P., Harding, M., \& Wegner, G. 1988, Nature, 336, 358

Weir, N., et al. 1993a, Astron. Soc. Pacific Conference Series, 43, 135

Weir, N., et al. 1993b, Astron. Soc. Pacific Conf. Series, 52, 39

Weir, N., et al. 1994, IAU Symp. 161, 205 eds. H. MacGillivray et al.

Weir, N., Djorgovski, S. \& Fayyad, U. 1995a, Astron.J., 110, 1

Weir, N., Fayyad, U. \& Djorgovski, S. 1995b, Astron.J., 109, 2401

Weir, N., et al. 1995c, Publ.Astron.Soc.Pacific, 107, 1243 